\title{
Les sigles français et polonais : entre noms propres et noms communs. L'impact de la valeur sémantique des sigles sur leur traduction
}

\author{
Polish and French acronyms: between a proper \\ and a common name. The influence of semantic values \\ of acronyms on their translation
}

\author{
Dorota Lajus \\ Université Mikołaj Kopernik, Pologne \\ dorotalajus@umk.pl
}

\begin{abstract}
Acronyms are lexical units that have been thoroughly explored in terms of their morphology or pronunciation. However, their semantic value has not been entirely determined yet. The present paper aims at investigating the manner in which a semantic value of an acronym, and particularly a difference between proper and common acronyms, influences techniques applied to translating them.

In order to achieve the aforementioned objective, on the basis of the cognitive theory of proper names, the authors have distinguished Polish and French acronyms being ergonyms as well as acronyms belonging to proper names and these designating item classification. The paper concerns the analysis of both Polish and French translations of ergonyms in foreign language press and French common acronyms in the Polish press. The performed analysis allows observing a tendency towards transferring acronymsergonyms that play the role of a rigid designator rather than full names in the target text. By contrast, the techniques of translating common acronyms differ, depending on whether they designate phenomena typical of the source culture or universal ones.
\end{abstract}

Keywords: acronym, common name, press discourse, proper name, translation technique

La siglaison, considérée comme processus de néologie lexicale, fait l'objet d'études approfondies d'ordre morphologique centrées sur les procédés de formation des sigles, et les classifications de ces lexèmes se font d'habitude en fonction de leur 
structure et prononciation. D'autres travaux touchent les questions de leurs flexion, orthographe ou encore de la dérivation à partir de sigles. Mais leur statut sémantique demande une analyse, car il reste ambivalent. D'un côté, les sigles assurent une identification immédiate des individus (organismes, services, programmes, etc.), ce qui les rapproche des noms propres ; de l'autre, certains d'entre eux font partie des appellations collectives désignant des classes d'objets et relèvent donc des noms communs. Le présent article a pour but d'observer si et dans quelle mesure la différence entre les sigles propres et les sigles communs a de l'impact sur les procédés de traduction appliqués.

\section{DÉFINITION DES SIGLES}

Pour ce travail, nous nous appuyons sur la définition du sigle proposée par Germain et Lapierre : « un signe abréviatif formé d'un assemblage de lettres ou groupes de lettres initiales [...] provenant d'un synthème discontinu d'usage fréquent et spécifique, assemblage généralement soumis à des contraintes d'ordre économique et/ou phonique »(Germain, Lapierre, 1988, p. 63). Cette conception large de la siglaison permet d'y adjoindre l'acronymie. Nous comprenons un acronyme comme « un groupe de mots raccourcis en conservant le début (le plus souvent la première syllabe) de chaque mot»(Calvet, 1980, p. 7). Le processus de siglaison s'opère donc généralement à partir de plus d'un mot et aboutit à la création d'un signe linguistique autonome qui concurrence sa source, c'est-à-dire son développement. Ceci le distingue d'une abréviation (c.-à-d., ex., pp. (fr.), tzn., np., str. (pl)) comprise comme une réduction uniquement graphique d'un lexème (Germain, Lapierre, 1988, p. 61), qui ne possède pas de réalisation orale distincte. En linguistique polonaise, les définitions des sigles (pl. skrótowce) sont parallèles. Ainsi dans les travaux de Jadacka (2010), Młodyński (1981) et Podracki (1999), on définit les sigles comme dérivés des noms d'institutions, d'entreprises, d'associations, etc., construits à partir des lettres ou des syllabes initiales des mots composants ces noms.

Calvet a observé que dans des échanges oraux certains sigles concurrencent les noms entiers des organismes à tel point que les locuteurs les utilisent et les comprennent correctement sans être capables de citer exactement leurs développements. Il en conclut qu'un sigle peut « avoir dans l'oralité une existence autonome, renvoyer directement à un signifié sans que la médiation de sa source ne soit nécessaire » (Calvet, 1980, p. 78). Percebois (2001, p. 629), qui a étudié le fonctionnement des sigles en français et anglais de spécialité, leur accorde une « fonction dénominative lapidaire » dans la mesure où d'un côté, ils dénomment et de l'autre, ils « facilitent la communication en synthétisant le concept considéré », répondant ainsi à la recherche du moindre effort. Etant donné que nombre de sigles français et polonais dénomment des organismes tels que des associations, comités, institutions, 
organisations administratives, sociétés commerciales et industrielles, établissements scolaires, partis politiques, syndicats, équipes de sport, etc. (Lajus, 2018, p. 98), ceci les rapproche des noms propres.

\section{LES SIGLES : ENTRE NOMS PROPRES ET NOMS COMMUNS}

Selon l'approche cognitive proposée par Jonasson (1994) et Leroy (2004), la fonction essentielle du nom propre est de nommer des individus et de fixer leur caractère unique :

A la différence des Nc qui, en vertu de leur sens, nous aident à regrouper des objets, des individus et des phénomènes ayant des propriétés en commun, les Npr nous permettent d'isoler des entités uniques et spécifiques, en nommant des particuliers perçus à l'intérieur des catégories établies (Jonasson, 1994, p. 16).

Le nom propre désigne donc directement un référent, il est « un désignateur rigide » dans le sens de Kripke (1972), et son rôle fondamental est identifiant et distinctif. Parmi les noms propres, d'après Jonasson, on peut distinguer ceux qui sont prototypiques, tels que les noms des personnes et des lieux, qui désignent un référent unique de manière apparemment exclusive et non descriptive et qui sont ressentis comme nomina propria de façon unanime. Mais on retrouve également des noms qui manifestent moins nettement ces caractéristiques typiques et se situent à la périphérie de la classe car ils sont descriptifs, possèdent plusieurs référents ou désignent des individus divers.

Leroy (2004, p. 9) reconnaît le caractère ambivalent des sigles, qui se placent entre les noms communs et les noms propres. D'après la typologie référentielle basée sur la nature de leur référent, les sigles propres peuvent être rattachés aux ergonymes (pl. ergonimy), c'est-à-dire aux noms de productions ou de découvertes humaines qui ont une réalité matérielle, telles que les entreprises, marques, établissements ou institutions. D'autres sigles propres font partie des praxonymes, autrement dit des noms « de réalisations ou de découvertes humaines non matérielles » (Leroy, 2004, p. 34), parmi lesquels Leroy cite des noms d'événements historiques et culturels, de maladies, de lois ou de théorèmes. Ces deux groupes correspondent à la catégorie des noms propres distinguée par A. Gałkowski (2007, p. 497), à savoir « les socioidéonymes » (pl. socjoideonimy), parmi lesquels le linguiste polonais classe les noms des organismes et institutions, donc des ergonymes, mais en plus les noms des idées, opérations, actions, initiatives, programmes, etc.

Comme le remarque Leroy, le sentiment que les ergonymes et les praxonymes appartiennent à la catégorie des noms propres est incertain. De plus, les noms d'organismes dont sont dérivés les sigles sont souvent descriptifs, composés de noms communs précisant la classe d'objets (société, institut, bibliothèque, etc.), 
d'adjectifs et de prépositions, leur sens est donc plus ou moins transparent. De surcroit, ils sont souvent introduits en français par un article, ce qui les place à la périphérie des noms propres :

En ce qui concerne les Npr constitués par des éléments descriptifs auxquels on associe normalement un concept, leur statut de $\mathrm{Npr}$ est plus périphérique. Ils se distinguent pourtant des Nc par la majuscule, le figement de leur formule et par le lien dénominatif qui les lie directement à un particulier (Jonasson, 1994, p. 41)

Ce qui fait donc que les noms comme Prawo i Sprawiedliwość ou Mouvement démocrate, Agence France Presse ou Krajowa Rada Sadownictwa sont traités comme noms propres, c'est leur fonction dénominative et du point de vue morphosyntaxique, leur caractère figé et les majuscules à l'écrit. Nous allons voir par la suite que les sigles remplissent encore plus ce caractère identifiant.

Toutefois, une étude comparative du fonctionnement des sigles français et polonais dans le discours de presse (Lajus, 2018) démontre une différence importante, car même si dans les deux langues la plupart des sigles servent à dénommer des organismes, en français nombreux sont les sigles lexicalisés que l'on retrouve dans les dictionnaires de langue, repertoriés comme des noms communs du fait qu'ils désignent des classes d'objets. Ils renvoient entre autres à des statuts de personnes, des objets de la vie quotidienne, des diplômes et niveaux d'éudes, des services médicaux, des salaires et allocations, des types de contrat, des secteurs d'activités, des quartiers, etc.

\section{ANALYSE DE LA TRADUCTION DES SIGLES DANS LA PRESSE}

Nous nous proposons d'observer les traductions des sigles français dans la presse polonaise et des sigles polonais dans la presse française, en poursuivant ainsi les recherches entamées par Skibińska $(2004,2005)$. Nous excluons de notre analyse les emprunts à l'anglais employés en français et polonais, sauf s'ils apparaissent dans le texte cible pour traduire des sigles de langue de départ.

Les exemples cités viennent de deux sortes de sources. La première, ce sont les textes parus dans Le Monde diplomatique ( $L M D$ ) et leurs traductions publiées dans l'édition polonaise de ce mensuel entre juillet 2009 et septembre 2018. Le deuxième groupe de documents est constitué des écrits parallèles, autrement dit, des textes rédigés en langues différentes mais appartenant au même genre discursif et se ressemblant par leur thématique et traits pragmatiques (Drescher, 2000, p. 68). Il s'agit d'articles de quotidiens nationaux d'opinion tels que Le Monde, Le Figaro, Rzeczpospolita, Gazeta Wyborcza $(G W)$ et de l'hebdomadaire Polityka. Les exemples viennent majoritairement des articles parus de mars 2017 à septembre 2018. Nous nous concentrons sur les textes d'une thématique générale d'actualité, celle des rubriques : politique et société. Ce choix nous permet d'étudier l'emploi des sigles dans les articles adressés à un large public. 
Dans notre étude, nous prenons en compte que la visée informative des textes de presse influe sur la manière de traduire les sigles, même si elle n'est pas la seule, car le discours journalistique est aussi persuasif (Charaudeau, 2006). Il est vrai également que les procédés de traduction dépendent des normes rédactionnelles qui peuvent varier d'un titre de presse à l'autre. Nonobstant, nous pouvons percevoir des analogies qui permettent de tirer des conclusions plus générales.

\subsection{La traduction des sigles propres}

Le premier type de sigles sont des ergonymes, autrement dit, des noms d'organismes. Ils remplissent une fonction dénominative lapidaire et dans une communication intralangue répondent à une loi de moindre effort en assurant une communication rapide à condition qu'ils soient largement connus. En revanche, au moment de la traduction, les sigles dénommant des organismes propres au pays d'origine ne possèdent pas leur correspondant dans le pays et la langue cible et comme note Ballard (2001, p. 41), deviennent souvent vides de sens. De ce fait, ils sont « porteurs d'étrangeté dans le texte cible » (Lewicki, 2000, p. 52 ; Borowczyk, 2012, p. 8) et les traducteurs ou rédacteurs deviennent des « médiateurs interculturels » dans la mesure où ils « cherchent à expliquer la réalité nouvelle inconnue au lecteur de leur texte (tel qu'il l'imaginent) » (Skibińska, 2004, p. 53).

En règle générale, dans notre corpus d'analyse, les sigles dénommant des organismes divers sont transférés dans leur forme originale. Il s'agit donc d'un report dans le sens de Ballard : « transfert intégral d'un nom propre du TD (texte de départ) dans le TA (texte d'arrivée) » (Ballard, 2001, p. 18). Dans la plupart des cas, lors du premier emploi dans un texte, ils sont accompagnés de la traduction en polonais de leur développement, parfois le nom entier français est reporté lui aussi. L'ordre dans lequel ils sont juxtaposés dans une phrase n'est pas fixe car parfois le sigle, parfois son étymon se retrouvent entre parenthèses ou en apposition. Ensuite, lorsque le sigle est mentionné dans la suite du texte, il est transféré dans sa forme originale sans développement ou ajouts explicatifs. Ce sont des procédés qui dominent nettement dans la traduction du Monde diplomatique. Ceci montre que le sigle remplit une fonction identifiante et reste un désignateur rigide encore plus qu'un nom entier abrégé par le sigle.

Parmi les sigles les plus fréquents sont les sigles dénommant les partis politiques. Leur première occurrence dans les textes du Monde diplomatique est presque systématiquement accompagnée de la traduction du nom du parti. Ainsi, pour les partis français, on trouve : PS (Partia Socjalistyczna), PCF (Francuska Partia Komunistyczna), UMP (Unia na rzecz Ruchu Ludowego), FN (Front Narodowy), RN (Zrzeszenie Narodowe), FI (Nieuległa Francja), NPA (Nowa Partia Antykapitalistyczna), etc. 
(1) S'appuyant exclusivement sur les cas des réfugiés climatiques et politiques, le candidat de La France insoumise entend « lutter contre les causes des migrations » (LMD, avril 2017).
Mélenchon, kandydat Nieuległej Francji (FI), zapowiada, że będzie « walczył z przyczynami migracji » (LMD, avril 2017).

Dans cet exemple, le sigle n'apparaît pas dans le texte original, mais il est employé comme sigle emprunté à la langue cible alors que le nom du parti est traduit en polonais. Le sigle sert donc d'« étiquette » utilisée pour identifier.

Dans les articles de presse qui ne sont pas des traductions, les sigles sont reportés, mais les stratégies de l'explicitation varient car on trouve les développements français, leurs traductions polonaises et de courtes gloses explicatives de nature diverse. Par exemple, le sigle REM est accompagné du nom entier du parti en français ou polonais (La République En Marche ! Republika w Marszu, Republika naprzód, Republiko Naprzód) et d'expressions synonymiques ou métonymiques diverses :

1. La République En Marche ! (REM), partia prezydenta Emmanuela Macrona, oraz jej koalicjant MoDem wygrały wybory parlamentarne we Francji (Polityka, juin 2017).

Pour les partis polonais mentionnés dans la presse française, on trouve entre autres : le PiS (Droit et justice), la PO (la Plate-forme civique), le KOD (Comité de défense de la démocratie), etc.

2. Vainqueur des élections d'octobre 2015 , le parti conservateur polonais Droit et justice (PiS) multiplie les démonstrations d'autoritarisme. [...] Le PiS, ce sont les seuls qui nous soutiennent, ils sont proches des gens. [...] Le 25 octobre 2015, le PiS remportait les élections parlementaires (Diète et Sénat) avec 37,6\% des suffrages, contre $24,1 \%$ pour les libéraux et $8,8 \%$ pour les populistes de Kukiz'15 (LMD, mars 2016).

On retrouve, dans les articles traduits, des noms de syndicats français : $C G T$ (Powszechna Konfederacja Pracy), CFDT (Francuska Demokratyczna Konfederacja Pracy), FSU (Jednościowa Federacja Zwiazkowa), SNES (Krajowy Zwiazek Zawodowy Pracowników Szkót Średnich), UNSA (Krajowa Unia Autonomicznych Związów Zawodowych), Medef (Ruch Przedsiębiorstw Francji). De nombreux sigles reportés dénomment des instituts de recherches tels que l'INSEE (Państwowy Instytut Statystyki i Badań Ekonomicznych), l'INED (Narodowy Instytut Badań Demograficznych), l'INSERM (Instytut Zdrowia i Badań Medycznych), le CNRS (Krajowy Ośrodek Badań Naukowych). D'autres dénomment des grandes écoles, des agences et offices nationaux ainsi que des associations, sociétés, etc. 
L'édition française du Monde diplomatique insère les sigles et les noms entiers des organismes juxtaposés presque systématiquement lors de leur première apparition dans le texte, même quand ils sont très connus. C'est pourquoi on observe certains cas, plus rares, où le sigle dans le texte français est accompagné du nom entier, alors qu'en polonais il n'est expliqué dans aucun fragment de l'article bien qu'il s'agisse d'une institution française :

(4) «Je sais avant la fin de l'émission sur quoi « Przed końcem emisji wiem, jak będzie va titrer l'AFP [Agence France-Presse] », brzmiał tytuł depeszy AFP », zapewnia s'enorgueillit-il (LMD, mars 2014). $\mathrm{z}$ dumą (LMD, avril 2014).

Le procédé du report de sigle et de la traduction du nom de l'institution est observé aussi dans d'autres titres de presse polonaise, aussi bien pour la traduction des institutions connues que pour celles qui sont mentionnées dans la presse polonaise plus accidentellement :

(5) Mówi o tym raport Generalnego Inspektoratu Policji (IGPN) zwanego „policją w policji” ( $G W$, août 2018).

(6) Według danych ogłoszonych przez OFPRA (Francuski Urząd ds. Uchodźców i Bezpaństwowców) roczna liczba cudzoziemców występujących o azyl we Francji w 2017 r. po raz pierwszy przekroczyła 100 tys. ( $G W$, janvier 2018).

(7) Stowarzyszenie Merów Francuskich (AMF) wydało „Vademecum świeckości” zbiór zaleceń $\mathrm{w}$ sprawie właściwych zachowań $\mathrm{w}$ razie zetknięcia się z problemami natury wyznaniowej ( $G W$, décembre 2017).

Ces exemples prouvent que les sigles cités fonctionnant comme des noms propres remplissent une fonction d'identification, de désignateur rigide dans la langue cible bien plus que les noms entiers envers lesquels les stratégies de traductions varient davantage. Il en est de même pour les sigles des institutions polonaises dans la presse française :

(8) Mais l'Institut polonais de la mémoire nationale (IPN), étroitement contrôlé par le pouvoir, a fait savoir dans un courrier qu'il souhaitait apporter "quelques modifications » à l'inscription de la plaque, dont le texte a été conçu par la Fondation Geremek (Le Monde, septembre 2018).

(9) Les modifications, déjà adoptées la semaine dernière par la chambre basse du Parlement, concernent la loi sur le Conseil national de la magistrature (KRS), celle sur les tribunaux de droit commun et celle sur la Cour suprême (Le Monde, avril 2018).

(10) Nous voulons de meilleures salaires pour la fonction publique, une hausse du salaire minimum, a déclaré aux journalistes Piotr Szumlewicz, un responsable de la centrale syndicale OPZZ, principal organisateur de la manifestation dont le mot 
d'ordre a été « Assez ! ». [...] OPZZ a jugé ces hausses insuffisantes (Le Figaro, septembre 2018).

(11) La Pologne a enregistré en 2017 environ 402.000 naissances, soit 20.000 de plus que l'année précédente, selon un communiqué de l'Office central des statistiques (GUS) (Le Figaro, mai 2018).

La fonction informative des textes favorise cet emploi de désignateur rigide, et le traducteur, qui est un médiateur, reporte tel quel dans la langue cible le sigle lié strictement à un élément de la réalité étrangère en utilisant aussi des procédés d'explicitation. La traduction des noms entiers, étant donné qu'ils sont souvent transparents car formés d'appellatifs, informe sur la nature de l'organisme, autrement dit, sur les classes d'objets auxquelles ils appartiennent : parti, confédération, société, association, institut, école, office, inspectorat, etc. Des épithètes ou des compléments nominaux qualifient ces noms (national, français, polonais, socialiste, syndical, de travail, etc.).

Toutefois les sigles sont accompagnés également d'adjonctions explicatives diverses, surtout si le nom entier n'explicite pas la classe d'objet à laquelle appartient l'organisme donné. Nous comprenons ici adjonction dans le sens proposé par Guidère : «L'ajout d'informations inexistantes sur l'original par le biais d'une explicitation ou d'une expansion, que ce soit dans le corps du texte, en note de bas de page ou encore dans le glossaire » (Guidère, 2010, p. 86). A titre d'exemple, nous citons l'extrait dans lequel le nom entier a été remplacé par une explicitation des traits du syndicat jugés comme pertinents ('le plus grand syndicat français') :

\begin{tabular}{|r|l|}
\hline (12) L'homme a transformé le cabinet & Człowiek, który przekształcił firmę anali- \\
d'expertise Secafi, proche de la Confé- & tyczną Secafi powiązaną z największym \\
dération générale du travail (CGT), & francuskim związkiem - CGT w [...] Gru- \\
en [...] groupe Alpha $(L M D$, mars 2017). & pę Alpha $(L M D$, mars 2017).
\end{tabular}

En s'appuyant sur les exemples cités, on peut conclure que le sigle propre se prête à être reporté dans le texte cible plus facilement que son développement et le concurrence ainsi en tant que désignateur rigide. Ceci aussi bien pour les noms d'organismes d'une grande notoriété que pour ceux qui sont peu connus dans la culture de la langue cible.

\subsection{La traduction des sigles communs}

Plusieurs sigles présents dans les articles de presse sont plus ou moins lexicalisés et répertoriés dans les dictionnaires de langue en tant que noms communs. Ce phénomène est plus caractéristique de la langue française, c'est pourquoi nous 
considérerons dans cette partie les traductions du français en polonais. Du point de vue de leur référence, nous diviserons ces sigles communs en deux groupes. Le premier est constitué des sigles renvoyant à des concepts, classifications, phénomènes propres à la socioculture française pour lesquels on ne trouve pas d'équivalents dans la société polonaise. Le deuxième groupe embrasse des sigles désignant des réalités qui ne sont pas attachées uniquement à la culture française.

\subsubsection{Les sigles désignant des réalités propres à la socioculture source}

Dans la presse analysée on retrouve notamment des sigles des termes administratifs et juridiques, par exemple des noms de dispositifs de la politique de la ville, de zones et de répartitions administratives, de statuts juridiques des personnes, d'impôts, d'allocations, de procédures juridiques et administratives, etc. On peut les considérer comme des emprunts terminologiques internes ou «emprunts à un technolecte » (Sablayrolles, 2000, p. 236) dans la mesure où le législateur ou l'administration introduisent une nouvelle notion, classification, procédure etc., et son nom est repris avec le sigle dans le discours de presse non spécialisée.

Pour illustrer ce phénomène, nous citons les sigles des programmes de politique de la ville et des classements administratifs de terrains urbains : $H V S, D S Q, Z U S, Z F U$. Dans les textes français, ces sigles accompagnent leurs étymons ou apparaissent seuls, en revanche, dans les traductions polonaises on note le transfert des sigles alors que les procédés de traduction des noms entiers ou d'explicitation du sens des sigles varient. Leur fonction dénominative est donc maintenue dans le texte cible.

(13) Théâtre régulier de révoltes urbaines, Vaulx-en-Velin a expérimenté depuis la fin des années 1970 tous les dispositifs de la politique de la ville : le programme « Habitat et vie sociale " (HVS), la convention de développement social des quartiers (DSQ), le contrat de ville, les zones urbaines sensibles (ZUS), les zones franches urbaines (ZFU)... Cette des banlieues » (LMD, mars 2017). ville symbolise à elle seule la «crise

W mieście tym, będącym areną nieustannych
rewolt, od końca lat 70 . wypróbowywano naj-
różniejsze pomysły miejskiej polityki, pro-
gramy takie jak: „mieszkalnictwo i życie
społeczne” (HVS), „konwencja o społecz-
nym rozwoju dzielnic” (DSQ), „miejskie
strefy wrażliwe” (ZUS), „miejskie strefy”
(ZFU). To miasto całe jest symbolem kry-
zysu przedmieść (LMD, mars 2017).

Les termes soulignés dans ce fragment, ont un statut ambivalent. Ainsi, le nom du programme Habitat et vie social est dans le texte français écrit en majuscules et entre guillemets, comme un titre, un praxonyme, alors que le nom du plan développement social des quartiers et des territoires urbains est rédigé en 
minuscules. Parmi ces sigles, seul ZUS est enregistré dans le Petit Robert 2018. Dans la traduction, tous ces noms sont traduits et mis entre guillemets, ce qui pourrait suggérer des noms propres. Quant aux sigles, ils sont cités tels quels.

Les deux fragments suivants montrent la préférence au report des sigles avec des formes d'explicitation changeantes, que ce soit le report du développement français ou sa traduction :

\begin{tabular}{|l|l|}
\hline (14) La nouvelle aire commerciale et la ZFU & $\begin{array}{l}\text { Nowa strefa handlowa i ZFU (miejska stre- } \\
\text { sont vantées par les élus comme la pro- } \\
\text { fa zwolnień podatkowych) przedstawiane } \\
\text { messe d'une ville dynamique, moderne, } \\
\text { où se rencontrent toutes les catégories } \\
\text { sociales }(L M D, \text { mars 2017). }\end{array}$ \\
& $\begin{array}{l}\text { nego, dynamicznego miasta, w którym żyją } \\
\text { ludzie z różnych warstw społecznych ( } M M D, \\
\text { mars 2017). }\end{array}$ \\
\hline
\end{tabular}

(15) Jedyną odpowiedzią było wzmocnienie środków bezpieczeństwa i stopniowe wprowadzanie polityki quartiers sensibles czy ZUS (zones urbaines sensibles) (LMD, février 2010).

L'extrait (16) vient d'un article dont le style est moins officiel. Le sigle ZEP y est non seulement reporté, mais en plus décliné, même s'il n'est pas emprunté par la langue polonaise et qu'il s'agit d'une attestation isolée :

(16) W 1981 roku stworzono kategorię ZEP - Zone d'éducation prioritaire (strefa edukacji priorytetowej). [...] Zapomnieli, że rodzice dzieci z ZEP-ów nie czytają z nimi książek. Całe życie uczyłam w ZEP-ach ( $G W$, mars 2017).

Le transfert des sigles des noms de prestations sociales et de salaires (RSA, RMI) permet de préserver la spécificité des solutions qui n'ont pas d'équivalents exacts dans la société polonaise :

(17) En France, un étranger ne peut toucher le revenu de solidarité active (RSA) que s'il bénéficie depuis au moins cinq ans d'un titre de séjour l'autorisant à travailler ( $L M D$, décembre 2013).

(18) Le montant du revenu inconditionnel devrait être au moins égal à celui de la prestation supprimée - comme l'actuel revenu de solidarité active (RSA) ou les bourses étudiantes (LMD, mai 2013).
Na przykład we Francji obcokrajowiec może otrzymać zasilek (RSA) tylko pod warunkiem, że przez co najmniej 5 lat miał pozwolenie na pobyt upoważniający do pracy zarobkowej ( $L M D$, janvier 2014).

Wysokość dochodu bezwarunkowego powinna być przynajmniej równa likwidowanym świadczeniom, np. obecnej zapomodze socjalnej dla osób, które nie pracują i nie mają żadnych dochodów, samotnie wychowujących przynajmniej jedno dziecko 


\begin{tabular}{|l|l|}
\hline & $\begin{array}{l}\text { lub takich, które pracują, ale mają niskie } \\
\text { dochody (Revenu de solidarité active, } \\
\text { RSA) (LMD, mai 2013). }\end{array}$ \\
\hline $\begin{aligned} \text { (19) Auparavant, et même s'il n'en est pas } \\
\text { resté grand-chose dans la mesure }\end{aligned}$ & $\begin{array}{l}\text { Myśl o tym, że społeczeństwo powinno } \\
\text { zapewnić swoim członkom środki do } \\
\text { finalement votée, l'idée que la société } \\
\text { przetrwania, przewijała się wcześniej }\end{array}$ \\
doit à ses membres les moyens de leur & W 1988 r. w parlamentarnych debatach \\
subsistance avait hanté les débats & przy okazji tworzenia zasiłku RMI przez \\
parlementaires autour de la création & rząd Michela Rocarda... (LMD, mai \\
du revenu minimum d'insertion & 2013). \\
(RMI) par le gouvernement de & \\
M. Michel Rocard, en 1988 (LMD, & \\
mai 2013). &
\end{tabular}

Dans les trois extraits, les traducteurs citent les sigles dans leur forme originale alors que le nom entier n'est reporté que dans l'exemple 18. Dans les fragments 17 et 19, le nom français est substitué. La substitution est comprise ici d'après Ballard comme « l'insertion de la définition (ou d'une forme d'explication) dans le texte à la place du terme d'origine " (Ballard, 2001, p. 114). Dans 17 et 19, elle s'effectue par emploi d'un hypéronyme désignant la classe d'objet à laquelle appartient l'élément de réalité dénommé par le sigle (zasitek 'allocation'). L'exemple 18 contient une adjonction explicative sous la forme d'une définition incluse dans la phrase.

Les sigles des impôts français sont repris également. Dans le premier exemple, $C S G$ est suivi du nom français et d'une glose explicative, dans le deuxième, étant donné que le nom de l'impôt est transparent et résume ces principes, le rédacteur polonais l'a traduit :

(20) To reakcja na podwyższenie CSG (contribution sociale généralisée) - podatku przeznaczonego $\mathrm{w}$ pierwszej mierze na zmniejszenie deficytu ubezpieczeń społecznych ( $G W$, mars 2018).

(21) Po pierwsze, nie przebrzmiały jeszcze echa największej tegorocznej zmiany w budżecie, to jest modyfikacji podatku solidarnościowego od wielkich majątków (ISF) (Polityka, juillet 2018).

Le sigle PACS s'est lexicalisé en donnant un nom masculin qui s'écrit en minuscules, il dénomme une institution juridique bien répandue en France. Ce sigle, loin d'être connu du large public en Pologne, apparaît dans la presse polonaise depuis des années, notamment lors des discussions publiques sur l'introduction des formes de partenariat enregistré en Pologne ou sous l'influence de l'actualité française.

(22) To jednak dość kłopotliwy alians, bo spora część świeckich przeciwników „małżeństwa dla wszystkich" (z prawem do adopcji) nie ma nic przeciwko związkom partnerskim PACS, które można zawierać we Francji od 1999 r. - PACS jest 
w porządku. Mniej niż 10 proc. par zawierających „cywilne umowy solidarności”, czyli PACS, to związki homoseksualne. PACS pozwala im na wspólne opodatkowanie, ale „małżeństwo dla wszystkich” daje, oprócz prawa do adopcji, m.in. rentę po partnerze, dodatkowe ułatwienia w dziedziczeniu (partnerzy w PACS muszą spisać testament, choć nie płacą podatku spadkowego) i prawo do cywilnych ceremonii ślubnych $(G W$, février 2013).

Dans cet article le transfert du sigle est accompagné de toute une panoplie de formes explicatives et synonymiques, toutefois les auteurs du texte ne cherchent pas à le substituer.

Ces quelques exemples cités traduisent la tendance générale observée dans notre corpus, à reporter dans leur forme originale les sigles dénommant des concepts propres à la société française. Ce transfert du sigle renvoyant à un référent français spécifique est accompagné de procédés explicatifs et de formes synonymiques diverses. Ces procédés de traductions sont donc les mêmes que pour la traduction des sigles d'organismes nationaux.

\subsubsection{Les sigles désignant des réalités non spécifiques de la culture source}

Les procédés de traduction des sigles désignant des réalités qui ne sont pas attachées uniquement à la socioculture française diffèrent de ceux que nous avons analysés jusqu'à présent.

Ainsi, les sigles des termes du domaine des services médicaux sont généralement traduits par des termes polonais correspondants sans report du sigle français. Ceci concerne aussi bien les termes souvent employés dans la presse générale que ceux qui sont moins connus des lecteurs.

Par exemple, le sigle $I V G$ (interruption volontaire de grossesse) introduit dans la langue française avec la loi Veil (1975), et employé couramment pour concurrencer avortement et finalement devenir son synonyme (Lopez Diaz, 2014) est traduit dans la presse polonaise avec des noms communs : aborcja, aborcja na żadanie, aborcja na życzenie, spędzenie płodu, usunięcie ciąży, zabieg przerywania ciąży, zabieg przerywania ciąży na żądanie, zabieg usunięcia ciązy, zabieg, etc.

\begin{tabular}{|c|c|}
\hline $\begin{array}{l}\text { 3) Alors que dans la plupart des pays } \\
\text { d'Europe l'IVG est majoritairement } \\
\text { considérée comme un droit acquis, les } \\
\text { Américains restent divisés sur le sujet } \\
\text { (LMD, décembre 2013). }\end{array}$ & $\begin{array}{l}\text { O ile w większości krajów europejskich } \\
\text { uważa się na ogół prawo do aborcji za } \\
\text { prawo nabyte, o tyle Amerykanie są w tej } \\
\text { kwestii bardzo podzieleni ( } L M D \text {, décembre } \\
\text { 2013). }\end{array}$ \\
\hline
\end{tabular}

Il en est de même avec les sigles DPI (diagnostic préimplantatoire) ou FIV (fécondation in vitro) : 
(24) Le DPI oblige à passer par une fé- W przypadku diagnostyki preimplantacyjcondation in vitro (FIV) pour pouvoir nej należy dokonać zapłodnienia in vitro, choisir entre plusieurs embryons. [...] umożliwiającego dokonanie wyboru spośród Quant aux embryons surnuméraires kilku embrionów. [...] Nadliczbowe emissus des FIV ... (LMD, juin 2009). briony wytwarzane przy in vitro... ( $L M D$, juillet 2009).

Les sigles des termes du domaine des affaires ayant un équivalent polonais ne sont pas reportés non plus. S'il existe un sigle polonais correspondant, il est employé, par exemple $P K B$ (produkt krajowy brutto) pour PIB (produit intérieur brut), sinon on emploie des notions équivalentes polonaises :

\begin{tabular}{ll|l|} 
(25) Dans ces conditions, pourquoi des & Dlaczego w tych warunkach pracownicy \\
entreprises choisiraient-elles des CDI à & mieliby wybierać umowę na czas nieokre- \\
«protection croissante » plutôt qu'une & ślony ,ze wzrastającą ochroną", a nie ko- \\
succession de CDD ? ( $L M D$, juillet & $\begin{array}{l}\text { lejne umowy na czas określony? ( } L M D, \\
\text { juillet 2016). }\end{array}$ \\
2016).
\end{tabular}

A l'intérieur de la catégorie des sigles communs, on retrouve des lexies françaises pour lesquelles on emploie dans les textes polonais soit des sigles anglais soit des équivalents polonais non sigliques. Ainsi $O N G$ est d'habitude traduit par organizacja pozarządowa ou bien remplacé par le sigle anglais $N G O$ ou son dérivé polonais, un néologisme $N G O s y$.

(26) Résume M. Juan Carlos Cárdenas, vétérinaire et directeur de l'ONG Centro Ecocéanos (LMD, septembre 2018).
Podsumowuje Juan Carlos Cárdenas, weterynarz i dyrektor organizacji pozarządowej $(L M D$, septembre 2018).

Le fragment suivant présente le sigle $O G M$ (organisme génétiquement modifié) rendu par son correspondant anglais $G M O$, mais l'on trouve aussi des traductions avec le terme polonais organizm zmodyfikowany genetycznie.

(27) Nous ne sommes pas fermement oppo- Nie jesteśmy całkowicie przeciwni GMO sés aux OGM $(L M D$, septembre 2017). $\quad$ (LMD, septembre 2017).

De même, on lit dans les textes polonais le sigle anglais MSM ou mężczyźni majacy kontakty seksualne z mężczyznami pour traduire $H S H$ (hommes ayant des rapports sexuels avec des hommes), AIDS pour SIDA, DNA pour ADN, VAT pour $T V A$, etc. 
(28) A l'échelle internationale, une grande W wymiarze światowym ogromnej więkmajorité des HSH restent hors de portée szości MSM nie obejmują żadne programy des programmes de prévention du sida zapobiegania AIDS (FDM, novembre 2012). (FDM, novembre 2012).

Ces sigles communs renvoient à des référents qui ne sont pas ancrés spécifiquement dans la socioculture française, c'est pourquoi ils ne sont pas repris dans le texte cible dans leur forme originale. Ils sont traduits en polonais avec des sigles polonais correspondants à condition que ceux-ci soient employés dans le discours des non spécialistes, dans certains cas avec des sigles anglais empruntés par le polonais et enfin avec des expressions non sigliques. Ce dernier procédé s'impose par cette différence au niveau du discours en polonais et en français qui fait que la tendance à économiser se réalise plus souvent par la siglaison dans la presse générale française. Dans le cas où le sigle français apparait plusieurs fois dans le même article, l'absence du sigle correspondant polonais impose aussi au traducteur et au rédacteur des stratégies d'évitement de la répétition formelle y compris une recherche des synonymes.

\section{CONCLUSION}

Les sigles entrant massivement dans les discours non spécialisés, les traducteurs y sont souvent confrontés, c'est pourquoi ils nécessitent des recherches approfondies. Après une observation des exemples de traduction de sigles dans la presse, on peut tirer la conclusion générale que l'opposition 'sigles propres / sigles communs', en corrélation avec l'opposition 'référent spécifique de la socioculture source / référent largement partagé', ont une influence sur les procédés de traduction choisis. Pour plus de clarté, nous proposons une présentation synthétique sous forme de tableau des procédés de traduction cités dans ce travail :

Tableau 1. Procédés de traduction des sigles cités

\begin{tabular}{|c|c|c|}
\hline Nature du sigle & Procédé de traduction du sigle & Procédé d'explicitation du sigle : \\
\hline \multirow[t]{4}{*}{ Sigles propres } & \multirow[t]{4}{*}{ Report (12) $100 \%$} & traduction du nom entier (8) $67 \%$ \\
\hline & & report du nom entier (1) $8 \%$ \\
\hline & & autre forme d'explicitation (4) 33\% \\
\hline & & sans aucune forme d'explicitation (1) $8 \%$ \\
\hline \multirow{3}{*}{$\begin{array}{l}\text { Sigles communs à référent } \\
\text { spécifique de la culture } \\
\text { source }\end{array}$} & \multirow[t]{3}{*}{ Report (13) $100 \%$} & traduction du nom entier (5) 38\% \\
\hline & & report du nom entier (3) $23 \%$ \\
\hline & & autre forme d'explicitation (8) $61 \%$ \\
\hline $\begin{array}{l}\text { Sigles communs à référent } \\
\text { largement partagé }\end{array}$ & $\begin{array}{l}\text { Substitution (9) } 100 \% \\
\text { - dont substitution par un sigle } \\
\text { anglais (3) } 33 \%\end{array}$ & \\
\hline
\end{tabular}


Nous pouvons donc d'abord affirmer que les sigles propres ergonymes qui dénomment des organismes nationaux de la culture source maintiennent leur fonction de désignateur rigide de manière plus systématique que leurs étymons, c'est-à-dire les noms entiers, d'habitude descriptifs, envers lesquels les procédés de traduction varient davantage.

En revanche, l'observation de la traduction des sigles communs, et pour cette catégorie l'on trouve dans la presse surtout des sigles français, a fait surgir l'importance d'un autre facteur lié à la nature du référent. Ainsi, les sigles désignant des réalités caractéristiques de la socioculture française sont reportés, d'autant plus que la fonction informative d'un texte favorise les stratégies de préservation de l'étrangéité afin de la faire découvrir aux lecteurs. Tout comme dans le cas des noms d'organismes, la tendance à traduire le sens et transférer le signifiant d'origine est plus visible pour le sigle que pour son étymon.

Par contre, les sigles français communs dont les référents font partie d'un bagage cognitif commun à plusieurs communautés linguistiques sont sujets à des substitutions par des équivalents de la langue cible.

La présente analyse démontre que bien qu'ils risquent d'être non signifiants pour les lecteurs cibles, les sigles reportés s'infiltrent dans le texte d'arrivée en tant qu'identificateurs d'un référent de la culture source et ceci d'une manière plus marquée que leurs développements. 


\section{BIBLIOGRAPHIE}

Ballard, M. (2001). Le nom propre en traduction. Paris : Ophrys.

Borowczyk, P. (2012). « Traduction des sigles dans le contexte audiovisuel - un dialogue réussi ?». Studia Romanica Posnaniensia, 39, 5-21. DOI : https://doi.org/10.14746/strop.2012.392.001.

Calvet, L.-J. (1980). Les sigles. Paris : PUF.

Charaudeau, P. (2006). «Discours journalistique et positionnements énonciatifs. Frontières et dérives ». Revue SEMEN 22. Énonciation et responsabilité dans les médias. URL : http://journals.openedition.org/semen/2793 (23.11.2018).

Drescher, M. (2000). « Pour une analyse contrastive des types de discours : l'exemple du faire-part de décès en français et en espagnol ». Studia Romanica Posnaniensia, 25/26, 67-80.

Gałkowski, A. (2007). « Socjoideonimy a chrematonimy - miejsce nazw organizacji i inicjatyw społecznych w dynamice onimicznej języka ». In : A. Cieślikowa, B. Czopek-Kopciuch, K. Skowronek (ed.), Nowe nazwy własne - nowe tendencje badawcze. Kraków : Wydawnictwo PANDIT, 495-508.

Guidère, M. (2010). Introduction à la traductologie : penser la traduction : hier, aujourd'hui, demain. Bruxelles : De Boeck.

Jadacka, H. (2010). « Skrótowce ». In : A. Markowski (ed.), Wielki słownik poprawnej polszczyzny. Warszawa : PWN, 1666-1668.

Jonasson, K. (1994). Le nom propre. Constructions et interprétations. Louvain-la-Neuve : Duclot.

Kripke, S. (1972). « Naming and Necessity ». In : D. Davidson, G. Harman (ed.), Semantics of Natural Language. Dordrecht / Boston : D. Reidel Publishing Company, 253-355.

Lajus, D. (2018). " Comparaison du fonctionnement des sigles français et polonais dans le discours de la presse ». Annales Universitatis Mariae Curie-Skłodowska, 36, 97-108. DOI : 10.17951/ff.2018.36.1.97-108.

Leroy, S. (2004). Le Nom propre en français. Paris : Ophrys.

Lewicki, R. (2000). Obcość w odbiorze przekładu. Lublin : Wydawnictwo Uniwersytetu Marii Curie-Skłodowskiej.

Lopez Diaz, M. (2014). "Deux variantes stylistiques dans le même contexte : le tabou et l'euphémisme ». In : L. Gaudin-Bordes, G. Salvan (ed.), Figures du discours et contextualisation. URL : http://revel.unice.fr/symposia/figuresetcontextualisation/index.html?id=1313.

Młodyński, J. (1981). «Skrótowce we współczesnym języku polskim ». In : H. Kurkowska (ed.), Wspótczesna polszczyzna. Warszawa : PWN, 156-186.

Percebois, J. (2001). « Fonctions et vie des sigles et acronymes en contextes de langues anglaise et française de spécialité ». Meta, 46 (4), 627-645. DOI : https://doi.org/10.7202/003821ar.

Podracki, J. (1999). Stownik skrótów i skrótowców. Warszawa : Wydawnictwo Naukowe PWN.

Sablayrolles, J.-F. (2000). La néologie en français contemporain. Examen du concept et analyse de productions néologiques récentes. Paris : Honoré Champion.

Skibińska, E. (2004). "Traduire en polonais les sigles utilisés dans la presse française ». In : M. Gouadec (ed.), Mondialisation - Localisation - Francophonie(s). Paris : La Maison du Dictionnaire, 49-54.

Skibińska, E. (2005). "Skrótowce w polskich przekładach francuskich artykułów prasowych ». In : M. Piotrowska (ed.), Język trzeciego tysiąclecia III, vol. 2 : Konteksty przektadowe. Kraków : Tertium, 171-180.

\section{RÉSEAUGRAPHIE}

URL : <https://www.monde-diplomatique.fr/> (26.11.2018).

URL : <https://www.monde-diplomatique.pl/> (26.11.2018). 
URL : <https://www.lemonde.fr/> (26.11.2018).

URL : $<$ http://www.lefigaro.fr/> (26.11.2018).

URL : <https://www.polityka.pl/TygodnikPolityka $>$ (26.11.2018).

URL : $<$ https://www.rp.pl/> (26.11.2018).

URL : $<$ http://wyborcza.pl/0,0.html $>$ (26.11.2018). 